\title{
An in silico analysis of dynamic changes in microRNA expression profiles in stepwise development of nasopharyngeal carcinoma
}

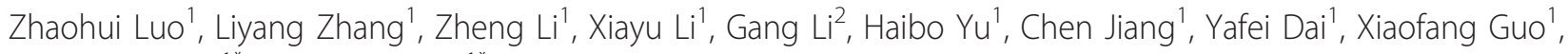
Juanjuan Xiang ${ }^{1 *}$ and Guiyuan Li $i^{*}$

\begin{abstract}
Background: MicroRNAs (miRNAs) are small non-coding RNAs that participate in the spatiotemporal regulation of messenger RNA (mRNA) and protein synthesis. Recent studies have shown that some miRNAs are involved in the progression of nasopharyngeal carcinoma (NPC). However, the aberrant miRNAs implicated in different clinical stages of NPC remain unknown and their functions have not been systematically studied.

Methods: In this study, miRNA microarray assay was performed on biopsies from different clinical stages of NPC. TargetScan was used to predict the target genes of the miRNAs. The target gene list was narrowed down by searching the data from the UniGene database to identify the nasopharyngeal-specific genes. The data reduction strategy was used to overlay with nasopharyngeal-specifically expressed miRNA target genes and complementary DNA (cDNA) expression data. The selected target genes were analyzed in the Gene Ontology (GO) biological process and Kyoto Encyclopedia of Genes and Genomes (KEGG) biological pathway. The microRNA-Gene-Network was build based on the interactions of miRNAs and target genes. miRNA promoters were analyzed for the transcription factor (TF) binding sites. UCSC Genome database was used to construct the TF-miRNAs interaction networks.
\end{abstract}

Results: Forty-eight miRNAs with significant change were obtained by Multi-Class Dif. The most enriched GO terms in the predicted target genes of miRNA were cell proliferation, cell migration and cell matrix adhesion. KEGG analysis showed that target genes were significantly involved in adherens junction, cell adhesion molecules, p53 signalling pathway et al. Comprehensive analysis of the coordinate expression of miRNAs and mRNAs reveals that miR-29a/c, miR-34b, miR-34c-3p, miR-34c-5p, miR-429, miR-203, miR-222, miR-1/206, miR-141, miR-18a/b, miR-544, miR-205 and miR-149 may play important roles on the development of NPC. We proposed an integrative strategy for identifying the miRNA-mRNA regulatory modules and TF-miRNA regulatory networks. TF including ETS2, MYB, Sp1, KLF6, NFE2, PCBP1 and TMEM54 exert regulatory functions on the miRNA expression.

Conclusions: This study provides perspective on the microRNA expression during the development of NPC. It revealed the global trends in miRNA interactome in NPC. It concluded that miRNAs might play important regulatory roles through the target genes and transcription factors in the stepwise development of NPC.

\footnotetext{
*Correspondence: xiangjj@csu.edu.cn; ligy@xysm.net

${ }^{1}$ Cancer Research Institute, Key Laboratory of Carcinogenesis and Cancer Invasion of Ministry of Education, Key Laboratory of Carcinogenesis of Ministry of Health, Central South University, 110 Xiangya Road, Changsha, Hunan, 410078, P.R. China

Full list of author information is available at the end of the article
} 


\section{Background}

Genetic and environmental factors are involved in the tumorigenesis and development of nasopharyngeal carcinoma (NPC). NPC is commonly diagnosed late due to vague early symptoms [1]. At first consultation, $70 \%$ of cases were diagnosed as cervical lymph node metastasis and $20-35 \%$ were diagnosed as long distant metastasis [2-4]. It is important to elucidate the cellular and molecular mechanisms of dynamic development of NPC. It is extremely necessary to identify the biomarkers and detect the high-risk factors in the progression of NPC.

microRNAs (or 'miRNAs', which are small noncoding RNA molecules) as post-transcriptional regulators have been a hotspot in research for their involvement in biological processes and tumour development $[5,6]$. They have been found to regulate genes involved in diverse biological functions, including development, differentiation, proliferation, and stress response. The dysregulation of miRNAs appears to play a crucial role in cancer pathogenesis where they exert their effect as oncogenes or as tumour suppressors [7]. A growing number of miRNAs have been implicated in carcinogenic process. A significant number of miRNAs have been mapped to cancer-associated genomic regions. Expression of the miRNA let-7 has been correlated with prognosis in lung cancer and found to regulate Ras in the same tumor [8]. Very recently, miR-10b has been shown to contribute to metastasis in breast cancer [9].

To date, several miRNAs have been shown to target specific mRNAs to regulate the progression of NPC. miR-216b [10], miR-218 [11], miR-26a/b [12,13], miR10b [14], let-7 [15], miR-141 [16], miR-200a [17] have been shown to have tumor suppressive functions in NPC. Not surprisingly, Epstein-Barr virus-encoded miRNAs have oncogenic properties [18-20]. It is well known that EBV infection has been identified as an essential factor in the carcinogenesis of NPC [21]. EBV-infection severely deregulates the miRNA profile of the host cell [22]. Several EBV encoded miRNAs were expressed at levels similar to highly abundant human miRNAs. EBVencoded miRNAs such as miR-BART1-5p, miRBART16, and miRBART17-5p, are intimately involved in processes leading to NPC $[23,24]$. The microRNA array has been performed by independent labs, identifying several differentially-expressed miRNAs in NPC [25-27]. The development of NPC is a multistage process, depending on spatial and temporal control of gene expression. Thus, it is unclear whether dysregulation of microRNA expression is an aberrant event that occurs during NPC progression. The dynamic regulatory roles of miRNA need to be explored. A key to understanding the role of miRNA is to determine when and where they are expressed [28]. Here, we studied the miRNA dynamic expression profiles in different clinical stages of NPC and NPC lymph node metastasis. The predicted miRNA target genes were compared to the cDNA expression in the Gene Expression Omnibus (GEO) (GSE12452). We found that a series of genes and miRNAs play an important role in the stepwise development of NPC.

Similar to protein-coding genes, the transcription of miRNAs is also regulated by transcription factors (TFs) $[29,30]$, an important class of gene regulators that act at the transcriptional level. The normal regulation of miRNAs by TFs is critical, and aberrant regulation of miRNAs by TFs can cause phenotypic variations and diseases [31]. Therefore, a TF-miRNA regulation database would be helpful for understanding the mechanisms by which TFs regulate miRNAs and then understanding their contribution to diseases.

In this study, the miRNA-gene interaction and transcription factor-miRNA interaction network were also described. Our study gives perspective on miRNAs expression in the stepwise development of NPC. It revealed the global trends in miRNA interactome in NPC.

\section{Methods}

\section{Patient samples and laser-capture microdissection}

Snap-frozen NPC biopsies were obtained from NPC patients and normal, healthy nasopharyngeal epithelial samples from biopsy-negative cases were used as control. The criteria of clinical staging of NPC samples was based on the 2008 staging system of NPC, which was established in 2008 according to NPC 92 and AJCC staging system [32,33]. NPC samples in clinical staging IIV were used (numbers I to IV, with IV having greater progression). Samples were collected from Xiangya Second Hospital, Central South University. The patients were informed about the sample collection and had signed informed consent forms. Collections and use of tissue samples were approved by the ethical review committees of Xiangya Second Hospital (Additional file 1). Laser capture microdissection was used to separate the cancer tissues from the normal tissues. Samples were first frozen-sectioned by using a LEICA CM 1900 cryomicrotome. Phase contrast images were acquired using LEICA CTR 6500 microscope.

\section{miRNA microarrays}

Total RNA was extracted using Trizol ${ }^{\circledR}$ reagent (Invitrogen) from samples. Two hundred nanograms (200 ng) of total RNA from each sample were used for the follow-up microarray. Poly(A) polymerase (PAP) was used to add a stretch of Poly-A tail to the 3' end of each sequence in total RNA. The Ambion Illumina TotalPrep 
RNA Amplification Kit was used to synthesize biotinylated cDNA. MicroRNA expression profiling kit contains primers for 1146 human miRNAs. The biotinylated cDNAs were hybridized with microRNA-specific oligonucleotides. The unbinding oligos were washed away and followed by the extension and ligation reaction. Polymerase Chain Reactions (PCR) were performed with fluorescently labelled universal primers, followed by hybridizing of the fluorescently labelled, single-stranded PCR products to capture beads. The fluorescent signals were then detected by Illumina's iScan System. All steps were performed according to Illumina's instructions manual.

\section{Bioinformatics analysis}

\section{Multi-Class Dif (RVM- $F$ test)}

Raw data from each array were analyzed using MultiClass Dif (RVM-F test) which is applicable to small sample size analysis for multiple groups. The RVM F-test was applied to screen the dynamic differentiallyexpressed genes. The detailed methods were applied as previously described [34-36].

\section{Gene Ontology (GO)}

The TargetScan database was used to predict the target gene of miRNAs. To understand the functions of predicted target genes, we used the ontology classification of genes based on gene annotation and summary information available through DAVID (Database for Annotation, Visualization and Integrated Discovery). The predicted target genes were assigned to functional groups based on molecular function, biological processes and specific pathways.

\section{TF-miR-net}

miRNAs sequence was mapped to the genome in the Sanger database. The Jemboss software was used to examine the alignment of sequences of pre-miRs and putative transcription factor binding sequences. A genome browser database was used to build the relationship of transcription factors and miRNAs network. An adjacency matrix was implemented in Java (programming language) according to the binding of pre-miRNAs and transcription factors. The network's core transcription factor is the most important centre with the biggest degree $[37,38]$. The Pearson correlation analysis [37] is used to measure the regulatory ability of transcription factors by calculating the correlation between transcription factors and the miRNAs.

\section{MicroRNA-gene-network}

The MicroRNA-Gene-Network was based on the interactions of miRNAs and target genes [39]. Twenty miRNAs of interest were built determined by pathways extracted from KEGG as primary nodes-networks. The significance of relationship of the miRNAs and target genes network was evaluated by the number of nodes in the network with degree greater than 10. In the MicroRNA-Gene-Network, the circle represents gene and the square represents MicroRNA, and their relationship was represented by one edge.

\section{Quantitative reverse transcription-polymerase chain reaction analysis}

Total RNA was extracted using Trizol ${ }^{\circledR}$ reagent (Invitrogen) from samples. The primers for RT-PCR to detect miRNA were designed based on the miRNA sequences provided by the Sanger Center miRNA Registry. The primers were synthesized and purified by the Shanghai Gene-Pharma Co. (Shanghai, China). RT reactions were performed by means of the iScript cDNA synthesis kit (Bio-Rad, Hercules, CA). Real-time PCR was performed on the BIO-RAD IQTM5 Multicolor Real-Time PCR detection System (Bio-Rad). The qPCR cycle was $98^{\circ} \mathrm{C}$ for $2 \mathrm{~min}$., 40 cycles of $95^{\circ} \mathrm{C}$ for $15 \mathrm{sec}$., $60^{\circ} \mathrm{C}$ for $30 \mathrm{sec}$. Final melt-curve analysis $\left(60^{\circ}-95^{\circ} \mathrm{C}\right)$ was included. The standard curve was produced with slopes at approximately $-3.32(\sim 100 \%$ efficiency); miRNA PCR quantification used $2^{\Delta \Delta \mathrm{ct}}$ method against the U6 for normalization. mRNA PCR quantification used $2^{\Delta \Delta \mathrm{ct}}$ method against the GAPDH for normalization. The data are representative of the means of three experiments.

RT-PCR primers:

miR-18a-F: TAAGGTGCATCTAGTGCAGATAG miR-18b-F: TAAGGTGCATCTAGTGCAGTTAG mR-141-F: TAACACTGTCTGGTAAAGATGG miR-149-F: TCTGGCTCCGTGTCTTCACTCCC miR-99a-F: AACCCGTAGATCCGATCTTGTG miR-99b-F: CACCCGTAGAACCGACCTTGCG miR-206-F: TGGAATGTAAGGAAGTGTGTGG miR-34b-F: CAATCACTAACTCCACTGCCAT miR-34c-3-F: AATCACTAACCACACGGCCAGG miR-34c-5-F: AGGCAGTGTAGTTAGCTGATTGC miR-29b-F: TAGCACCATTTGAAATCAGTGTT miR-29c-F: TAGCACCATTTGAAATCGGTTA miR-429-F: TAATACTGTCTGGTAAAACCGT miR-32-F: TATTGCACATTACTAAGTTGCA miR-181c-F: AACATTCAACCTGTCGGTGAGT U6-F: ATTGGAACGATACAGAGAAGATT U6-R: GGAACGCTTCACGAATTTG ATM-F: GGACAGTGGAGGCACAAAAT ATM-R: GTGTCGAAGACAGCTGGTGA BCL2-L2-F: TCGCCCTGTGGATGACTGA BCL2-L2-R: CCAGGAGAAATCAAACAGAGGC CDH1-F: CGAGAGCTACACGTTCACGG CDH1-R: CGAGAGCTACACGTTCACGG E2F3-F: CACTTCCACCACCTCCTGTT E2F3-R: TGACCGCTTTCTCCTAGCTC YY1-F: CAAGAAGTGGGAGCAGAAGC YY1-R: CTGCCAGTTGTTTGGGATCT MYB-F: ACAGTCATTTGATGGGTT 
MYB-Re TCTCGGTTGACATTAGGA

Dcier-Fe AAGGAAGCTGGCAAACAAGAe

Dcier-R: AAAACGAACCACCAAGTTGCe

Smad2-F: CGAAATGCCACGGTAGAAAT

Smad2-R: CCAGAAGAGCAGCAAATTCC

KLF6-F: CACGAGACCGGCTACTTCTC

KLF6-R: CGGATTCCTCCTTTTTCTCC

GAPDH-F: GAGTCAACGGATTTGGTCGT

GAPDH-R: TTGATTTTGGAGGGATCTCG

\section{Results}

1. miRNA expression profile in the stepwise development of NPC

In order to identify miRNAs associated with the stepwise development of NPC, miRNA microarrays were performed. We analyzed the temporal patterns of miRNAs expression profiles during the development of NPC. Samples were taken from a range of tumors of different stages. 6 cases of normal, 4 cases of each stage I or II, III, IV and 4 cases of lymph node metastasis were taken. The microdissection was performed with Methyl Green staining to separate tumor cells to non-tumor cells (Figure 1A). The Illumina microRNA Expression Profiling Assay was performed. The raw data of miRNA array can be downloaded from the National Center for Biotechnology Information-Gene Expression Omnibus (GEO) (GEO:GSE32906). From MicroRNA profiling, after separating signal from noise, we obtained $766 \mathrm{miR}$ NAs. Microarray data showed that 48 miRNAs were differentially expressed with significant change in tumor samples compared to normal samples (Multi-class Dif multiple comparison test RVM-F test, $\mathrm{P}<0.05$, FDR $<$ 0.05) [34-36]. Differentially expressed miRNAs between various stages of NPC and normal nasopharyngeal epithelia were clustered by Cluster3.0, as shown in the Figure 1B. Using the dendrogram-based methods for Clustering, the samples can be further separated into five subgroups on hierarchical clustering based on their similar expression patterns, which were correlated with the NPC clinic stages. The results showed that the expression pattern of miRNAs from the different clinical stages can be distinguished from each other (Figure 1B). We identified differentially-expressed miRNAs between clinical stages, in which 12 miRNAs differentially expressed between stage I-II and normal, 15 miRNAs between stage III and normal, 20 miRNAs between stage IV and normal, 37 miRNAs between lymph node metastasis and normal, as shown in Table 1. Our study revealed dynamic miRNA expressions, which were classified into 6 different patterns (Figure 1C). In pattern 1, miRNA expressions gradually decreased during the development of NPC. In pattern 5, miRNA expressions gradually increased during the development of NPC. In pattern 6, miRNA expressions dramatically increased in the lymph node metastasis.

These results suggested that these miRNAs might play important roles in the stepwise development of NPC.

\section{Target gene prediction of miRNAs and gene function analysis}

Putative target genes of 48 differentially expressed miRNAs were searched with online algorithms for miRNA target prediction (TargetScan). More than a thousand target genes were predicted for the $48 \mathrm{miR}$ NAs. The predicted target gene lists were narrowed down by searching the data from ftp://ftp.ncbi.nih.gov/ repository/UniGene/ to find the nasopharyngeal- specific genes.

We compared gene expression profiles of NPC biopies and miRNA expression profiles done above. The expression data (GSE12452) from biopsies of NPC and nonmalignant controls were downloaded from the National Center for Biotechnology Information-Gene Expression Omnibus (GEO). Putative target genes of miRNAs differentially expressed in NPC biopsies, which was consistent with the miRNA expression were shown in Table 2. We found that such as miR-29c and miR-34c-5 were down regulated, their target gene NDST1 and MMP2 were upregulated in NPC, miR-1/206 and their target CDH1, SMAD4, PDCD10, TGFBR3 are also consistent to each other, miR-18a/b and their target ATM and Samd4 also showed the coordinate expression. After microRNAs target filtering, the miRNAs which regulate the development of NPC through target gene regulation may be narrowed down. The selected genes were analyzed in the context of Gene Ontology (GO) biological process and Kyoto Encyclopedia of Genes and Genomes (KEGG) biological pathway using the molecular annotation. To assess the function of the predicted target gene, we evaluated the frequency of specific gene ontology terms among the predicted nasopharyngeal specific target genes of the 48 miRNAs using DAVID. The most enriched GO terms in the predicted targets genes of miRNA were cell proliferation, cell migration and cell matrix adhesion (Table 3). The predicted target genes were also analyzed by Kyoto Encyclopedia of Genes and Genomes (KEGG). As shown in Table 4, these target genes were significantly involved in adherens junction, pathway in cancer, cell adhesion molecules, p53 signaling pathway et al. It demonstrated that miRNAs with significant change are involved in regulation of target genes related to the development of NPC. These miRNA:gene interactions were built into a bipartite network (the miRNAome) (Figure 2). 


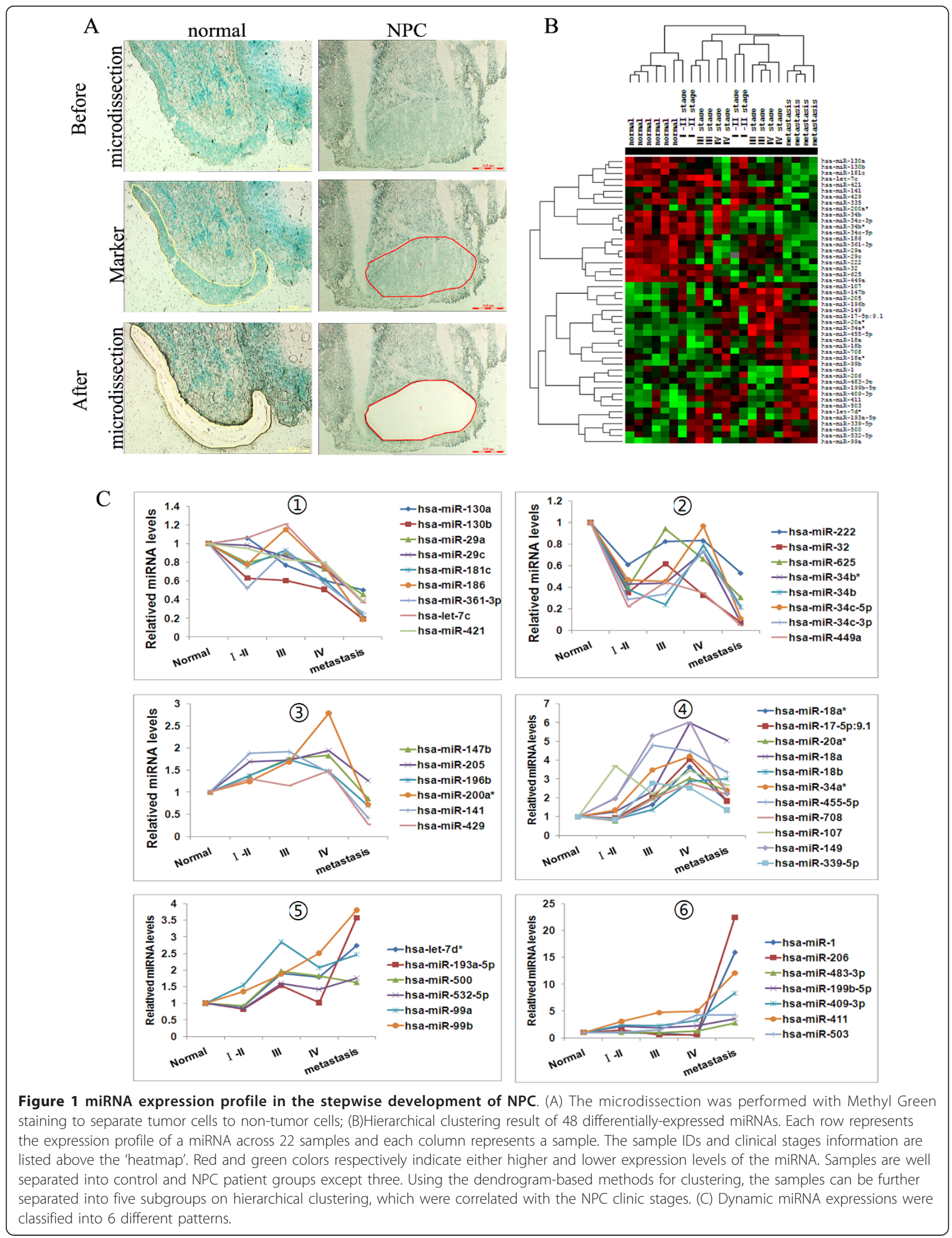


Table 1 The aberrantly expressed miRNAs in the different multi-stages of NPC

\begin{tabular}{|c|c|c|}
\hline Stage & Down-regulated miRNAs & Up-regulated miRNAs \\
\hline$|-| \mid$ & $\begin{array}{l}\text { hsa-miR-449a, hsa-miR-34c-3p, hsa-miR-32, hsa-miR-34b, hsa-miR- } \\
\text { 625, hsa-miR-34b* hsa-miR-34c-5p }\end{array}$ & $\begin{array}{l}\text { hsa-miR-455-5p, hsa-miR-199b-5p, hsa-miR-409-3p, hsa-miR-411, hsa- } \\
\text { miR-107 }\end{array}$ \\
\hline III & $\begin{array}{l}\text { hsa-miR-34b, hsa-miR-34c-3p, hsa-miR-34b*, hsa-miR-449a, hsa-miR- } \\
\text { 34c-5p }\end{array}$ & $\begin{array}{l}\text { hsa-miR-17-5p:9.1, hsa-miR-107, hsa-miR-409-3p, hsa-miR-18a, hsa- } \\
\text { miR-339-5p, hsa-miR-99a, hsa-miR-34a*, hsa-miR-411, hsa-miR-455- } \\
\text { 5p, hsa-miR-149 }\end{array}$ \\
\hline IV & hsa-miR-32, hsa-miR-449a, & $\begin{array}{l}\text { hsa-miR-99a, hsa-miR-199b-5p, hsa-miR-99b, hsa-miR-339-5p, hsa- } \\
\text { miR-708, hsa-miR-200a*, hsa-miR-18b, hsa-miR-20a*, hsa-miR-409-3p, } \\
\text { hsa-miR-107, hsa-miR-18a*, hsa-miR-17-5p:9.1, hsa-miR-34a*, hsa-miR- } \\
\text { 503, hsa-miR-455-5p, hsa-miR-411, hsa-miR-18a, hsa-miR-149 }\end{array}$ \\
\hline Metastasis & $\begin{array}{l}\text { hsa-miR-449a, hsa-miR-32, hsa-miR-34b*, hsa-miR-34c-5p, hsa-miR- } \\
\text { 186, hsa-miR-130b, hsa-miR-34c-3p, hsa-miR-34b, hsa-miR-181c, } \\
\text { hsa-miR-361-3p, hsa-miR-429, hsa-miR-625, hsa-miR-335, hsa-let-7c, } \\
\text { hsa-miR-29c, hsa-miR-421, hsa-miR-141, hsa-miR-29a }\end{array}$ & $\begin{array}{l}\text { hsa-miR-708, hsa-miR-149, hsa-miR-20a*, hsa-miR-34a*, hsa-miR-99a, } \\
\text { hsa-miR-107, hsa-let-7d*, hsa-miR-483-3p, hsa-miR-18b, hsa-miR-455- } \\
\text { 5p, hsa-miR-199b-5p, hsa-miR-193a-5p, hsa-miR-99b, hsa-miR-503, } \\
\text { hsa-miR-18a, hsa-miR-409-3p, hsa-miR-411, hsa-miR-1, hsa-miR-206 }\end{array}$ \\
\hline
\end{tabular}

\section{Validation of expression of microRNA and their target genes}

As mentioned above, we integrated the miRNA expression profile and cDNA expression profile. We narrowed down the candidate miRNA list which may play important roles on the development of NPC. To confirm microarray data, real-time RT-PCR experiments were conducted using specific primers for 15 miRNAs (miR206, miR-141, miR-149, miR-34b, miR-34c-3, miR-34c5, miR-18a, miR-18b, miR-99a, miR-99b, miR-429, miR32, miR-181c, miR-29b and miR-29c) in 38 NPC cases and 10 normal nasopharyngeal epithelial tissue cases. We confirmed the down-regulation of the miR-34 family (miR-34b, miR-34c-3, miR-34c-5, miR-429) and up-regulation of miR-17-92 (miR-18a/b) cluster. The dynamic expression patterns obtained with real-time RT-PCR were consistent with the microarray results (Figure 3). Furthermore, we also performed the real-time PT-PCR to evaluate the expression target genes. As shown in Figure 4, the expression of the selected target genes of the miRNAs showed the reverse correlation with the miRNA expression. We found that target genes such as CDH1 (miR-1/206), ATM (miR-18a/b), KLF6 (miR-18a/ $\mathrm{b}$ and $\mathrm{miR}-181 \mathrm{c}), \operatorname{Smad} 2(\mathrm{miR}-18 \mathrm{a} / \mathrm{b}, \mathrm{miR}-1 / 206$ and miR-149), Dicer(miR-18a/b) were down expressed with the development of NPC, while BCL2L2 (miR-29a/b/c and miR-203), E2F3(miR-34b/c and miR-429), ETS2 (miR-429), MYB(miR-429) and YY1 (miR-29a/b/c and $\mathrm{miR}-34 \mathrm{~b} / \mathrm{c}$ ) were overexpressed during the development of NPC.

\section{The miRNA transcriptional network for NPC development}

Although the expression of miRNAs and their targets is often highly correlated, anti-correlations exist because of miRNA feedback loops and upstream regulators,

Table 2 Putative target genes of differentially expressed miRNAs in NPC biopsies, which was consistent with the mRNA expression

\begin{tabular}{|c|c|c|c|}
\hline \multicolumn{2}{|c|}{ miRNA microarray } & \multicolumn{2}{|l|}{ cDNA microarray } \\
\hline miRNA & Expression & Target gene & expression \\
\hline hsa-miR-1/206 & up & API5, PCDH17, SMAD2, MTSS1, PDCD10, PDCD4, SMAD4 & down \\
\hline hsa-miR-141 & up & PDCD4, MTSS1, ARHGAP24, SIAH1, PTEN & down \\
\hline hsa-miR-149 & up & TP63, SMAD2, CNTNAP2 & down \\
\hline hsa-miR-18a/b & up & $\begin{array}{c}\text { DICER1, GIGYF2, STK4, RABGAP1, NOTCH2, NEDD9, ATM, SMAD2, NAV1, FOSL2, CAMKK2, STK4, BTG3, } \\
\text { UBE2Z, IRF2, LIN54, HIF1A, RABGAP1, CCDC88A, ETV6, CCND2 }\end{array}$ & down \\
\hline hsa-miR-205 & up & CADM1, MORF4L2, PTEN, SMAD1, SMAD4, TP53BP2 & down \\
\hline hsa-miR-544 & up & AKT2, CADM1, CDH1, SMAD4 & down \\
\hline hsa-miR-99a/b & up & BMPR2, IGF1R, THAP2 & down \\
\hline hsa-miR-203 & down & BCL2L2, ESR1, HBEGF, HBP1, HSPB8, IGFBP5, ITGA2, LPP, MAP3K1 MKL2, MLLT4, SOCS3 & up \\
\hline hsa-miR-222 & down & ESR1, ITGB8, MBD2, MIA3, MLL, SOCS3, TET2 & up \\
\hline hsa-miR-29a/c & down & BCL2L2, HBEGF, HBP1, HSPG2, ITGB1, LAMC2, LTBR, MIB1, MLF1, MMP2, NDST1, SVEP1 & up \\
\hline hsa-miR-34b & down & BTRC, CTNNA1, CUL3, CYLD, ESR1, HIPK1, ITGA2, ITM2B, MKL2, MXD1, PAPD5, PPP1CB, SMAD3 & up \\
\hline hsa-miR-34c-3p & down & DCBLD2, FOXN3, IKZF1, NPTN PAFAH1B1, USP10, YY1 & up \\
\hline hsa-miR-34c-5p & down & $\begin{array}{r}\text { ARHGAP1, ARHGEF3, BCL11B, C16orf5, CNTNAP1, FOXN3, FUT8, IL6R, ITGB8, ITSN1, JAG1, MLL2, NDST1, } \\
\text { NOTCH2, NPNT, PPFIA1, PTPRM, PVRL1, SERPINE1, VCL, YY1 }\end{array}$ & up \\
\hline hsa-miR-429 & down & MIB1, MLL5, NDST1, RHOA, RND3 & up \\
\hline
\end{tabular}


Table 3 GO Functional Annotation of the target genes

\begin{tabular}{ccccc}
\hline Term & Count & \% & PValue & FDR \\
\hline & up-regulated target gene GO functional annotation & & \\
GO:0022610 biological adhesion & 19 & 2.496715 & $2.93 \mathrm{E}-09$ & $4.71 \mathrm{E}-06$ \\
GO:0007155 cell adhesion & 19 & 2.496715 & $2.87 \mathrm{E}-09$ & $4.60 \mathrm{E}-06$ \\
GO:0010941 regulation of cell death & 17 & 2.233903 & $1.04 \mathrm{E}-06$ & 0.00166506 \\
GO:0043067 regulation of programmed cell death & 17 & 2.233903 & $9.88 \mathrm{E}-07$ & 0.001585717 \\
GO:0042981 regulation of apoptosis & 17 & 2.233903 & $8.67 \mathrm{E}-07$ & 0.0013906 \\
GO:0042127 regulation of cell proliferation & 17 & 2.233903 & $6.52 \mathrm{E}-07$ & 0.001046319 \\
GO:0007049 cell cycle & 16 & 2.102497 & $2.94 \mathrm{E}-06$ & 0.00471089 \\
GO:0051270 regulation of cell motion & 10 & 1.31406 & $3.26 \mathrm{E}-07$ & $5.23 \mathrm{E}-04$ \\
GO:0040012 regulation of locomotion & 10 & 1.31406 & $3.12 \mathrm{E}-07$ & $5.01 \mathrm{E}-04$ \\
GO:0030334 regulation of cell migration & 10 & 1.31406 & $1.05 \mathrm{E}-07$ & $1.69 \mathrm{E}-04$ \\
GO:0042127 regulation of cell proliferation & 13 & 2.901786 & $4.73 \mathrm{E}-07$ & $7.26 \mathrm{E}-04$ \\
GO:0016265 death & 12 & 2.678571 & $1.68 \mathrm{E}-06$ & 0.00257469 \\
GO:0008219 cell death & 12 & 2.678571 & $1.57 \mathrm{E}-06$ & 0.002405587 \\
GO:0012501 programmed cell death & 11 & 2.455357 & $2.87 \mathrm{E}-06$ & 0.004409001 \\
GO:0006915 apoptosis & 11 & 2.455357 & $2.52 \mathrm{E}-06$ & 0.003858936 \\
\hline
\end{tabular}

including transcription factors. Except for the above miRNA-target genes network, the TF-miRNA transcriptional interactions need to be elucidated. The predicted transcription factors binding to the miRNAs with the degree greater than 10 were shown in Table 5. Among them, 7 transcription factors were found differentially expressed in clinical stage I-II; 8 in stage III; 14 in stage IV and 21 in lymph node metastasis, compared to the

Table 4 The KEGG-PATHWAY analysis of the target genes

\begin{tabular}{cccc}
\hline Term & Count & $\%$ & PValue \\
\hline up-regulated target gene KEGG- & & & \\
PATHWAY & & & \\
hsa04520:Adherens junction & 7 & 0.9198423 & $5.40 \mathrm{E}-06$ \\
hsa04510:Focal adhesion & 7 & 0.9198423 & 0.001138399 \\
hsa04512:ECM-receptor interaction & 5 & 0.6570302 & 0.001552176 \\
hsa04514:Cell adhesion molecules & 5 & 0.6570302 & 0.007927108 \\
(CAMs) & & & \\
hsa04810:Regulation of actin & 6 & 0.7884363 & 0.008917732 \\
cytoskeleton & & & \\
hsa05200:Pathways in cancer & 7 & 0.9198423 & 0.012815044 \\
hsa04530:Tight junction & 4 & 0.5256242 & 0.047009212 \\
hsa04120:Ubiquitin mediated & 4 & 0.5256242 & 0.049654282 \\
proteolysis & & & \\
down-regulated target gene KEGG-PATHWAY & & \\
hsa05200:Pathways in cancer & 8 & 1.7857143 & $1.12 \mathrm{E}-04$ \\
hsa04115:p53 signaling pathway & 4 & 0.8928571 & 0.001901548 \\
hsa04520:Adherens junction & 4 & 0.8928571 & 0.002717119 \\
hsa05210:Colorectal cancer & 4 & 0.8928571 & 0.003481217 \\
hsa04350:TGF-beta signaling pathway & 4 & 0.8928571 & 0.003845193 \\
hsa04110:Cell cycle & 4 & 0.8928571 & 0.010537531 \\
hsa04310:Wnt signaling pathway & 4 & 0.8928571 & 0.017542982 \\
hsa04510:Focal adhesion & 4 & 0.8928571 & 0.036959365 \\
\hline & & &
\end{tabular}

normal samples. Seven TFs including ETS2, MYB, Sp1, KLF6, NFE2, PCBP1 and TMEM54 were shown in the middle of network, regulating most of the selected miRNAs (Figure 5). The larger triangles represent the more miRNAs which are regulated. The numbers of miRNA regulated by TF were represented by degrees. These transcription factor-miRNA interactions were built into a network.

We further investigated the TF-miRNAs-target genes feedback loop by integrating the TF-miRNA networks and miRNA-target genes networks. The possible regulatory feedback loops were shown in Table 6. These transcription factors may repress their own translation through induction of miRNAs as a negative regulatory feedback loop. This strategy combines paired expression profiles of TF, miRNAs and mRNAs in computational target predictions.

\section{Discussion}

While the process events that result in NPC remain unclear, global transcriptome analysis, including miRNAome $[40,41]$, is a useful tool to investigate dynamic change of molecular networks between different clinical stages. Although the microRNA array has been performed by two independent labs $[25,26]$, the dynamic miRNA expression during the development of NPC remains unknown. NPC is a multistage process that usually takes decades to develop. It is necessary to investigate the molecular events during the process. In our study, samples from different clinical stages of NPC were analyzed by the microRNA array. Microdissection was performed to ensure the purity of cancer tissues. Usually microarray techniques provide a valuable way of 


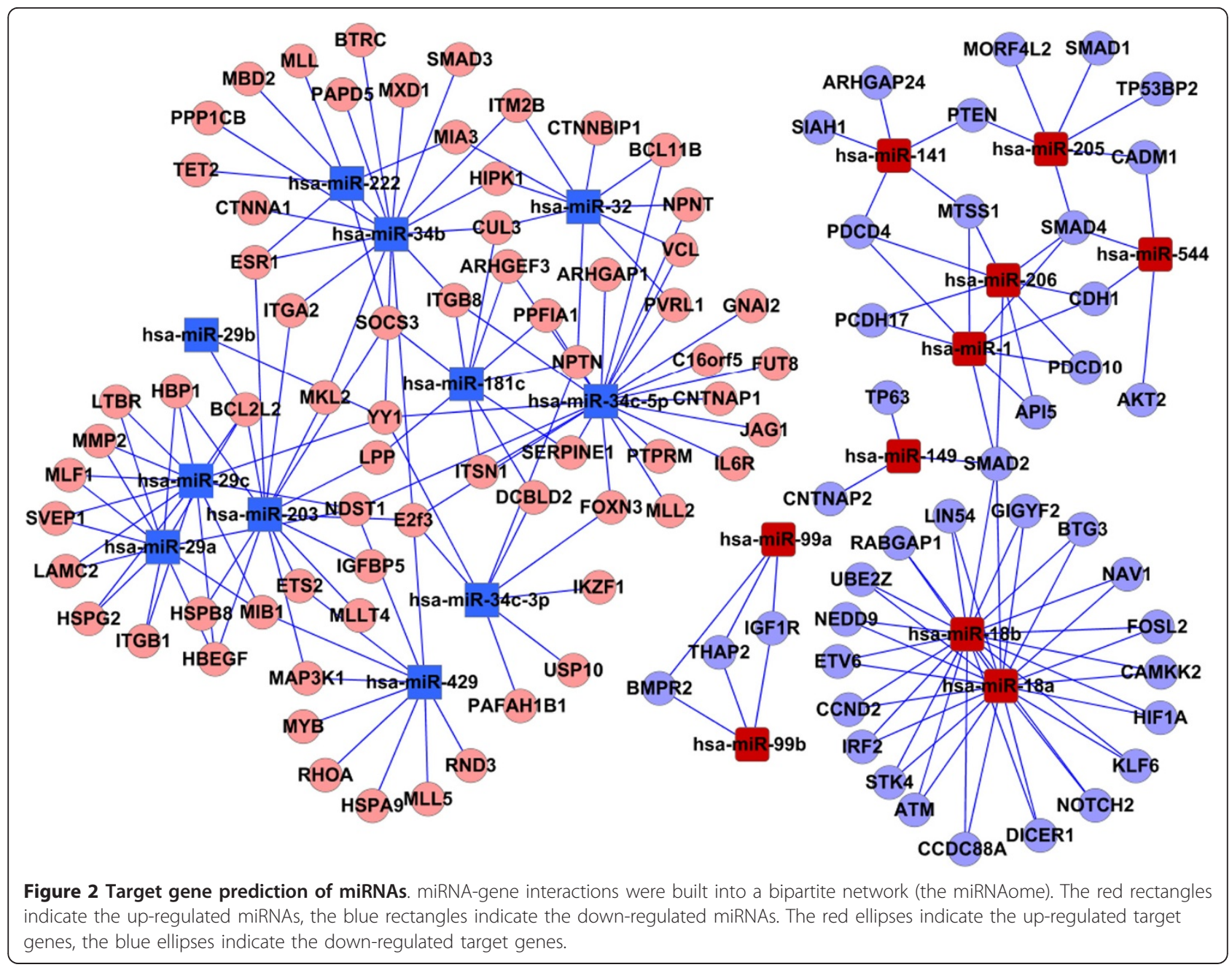

characterizing the molecular nature of disease but the expense and limited specimen availability often lead to studies with small sample sizes. This makes accurate estimation of variability difficult. Since variance estimates made on a gene-by-gene basis will have few degrees of freedom, the assumption that all genes share

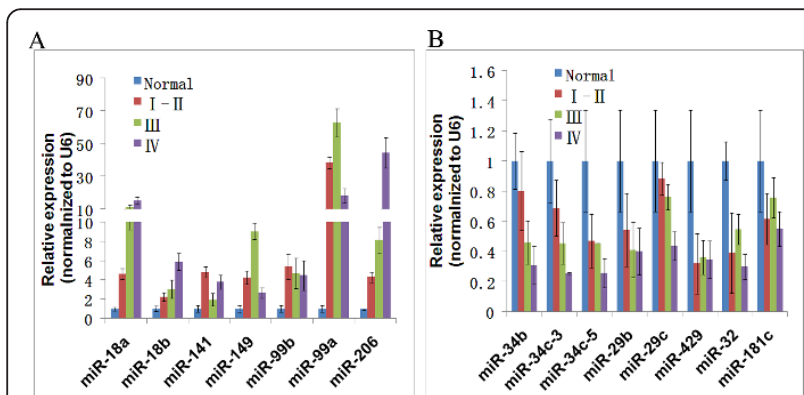

Figure 3 The expression miRNAs were verified by real-time PCR in 38 NPC cases and 10 normal. (A) the up-regulated miRNAs; (B) the down-regulated miRNAs. equal variance is unlikely to be true. To solve this problem, in this study, Multi-Class Dif was used which is applicable to the small sample sizes and we found that 48 miRNAs were differentially expressed in the four development stages and lymph node metastasis.

In our study, we present a global relative expression analysis of miRNAs in NPC. miRNAs with similar

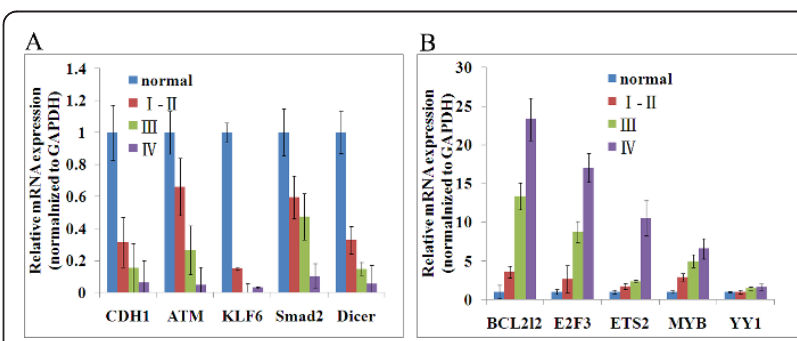

Figure 4 The expression of corresponding target genes of miRNAs were verified by real-time PCR in 38 NPC cases and 10 normal. (A) representative target genes of upregulated miRNAs; (B) representative target genes of downregulated miRNAs. 
Table 5 The predicted transcription factors binding to the $\mathbf{4 8}$ aberrant miRNAs with the degree bigger than 10

\begin{tabular}{ll}
\hline Stage & degree $\geq 10$ \\
\hline I-II & ETS2, MYB, Sp1, KLF6, NFE2, PCBP1, TMEM54 \\
III & ETS2, MYB, KLF6, SP1, PCBP1, TMEM54, YY1, NFE2 \\
IV & ETS2, MYB, NFE2, PCBP1, APP, KLF6, SP1, TMEM54, YY1, RUNX2, TFCP2, TEAD2, NR3C1, TFAP2A \\
Lymph node & ETS2, MYB, NFE2, SP1, YY1, KLF6, TMEM54, ESR1, PCBP1, APP, TEAD2, RUNX2, TFCP2, TFAP2A, MAZ, NR3C1, TBP, WT1, MYC, \\
metastasis & AP1S1, USF1 \\
\hline
\end{tabular}

expression profiles are clustered together in 6 patterns by cluster 3.0. In pattern 1, miRNA expressions gradually decreased during the development of NPC, which may function as a tumor suppressor gene, such as miR$29[42,43]$. In pattern 5, miRNA expressions gradually increased during the development of NPC, such as miR$18 \mathrm{a} / \mathrm{b}$. miR-18a/b was clustered together with miR-17$92[44,45]$ according to the similar expression profiles, which were induced by c-myc and reported to promote cell differentiation and proliferation. In pattern 6 , such as miR-206 [46] and miR-1 [47], miRNA expressions dramatically increased in the lymph node metastasis. It indicated that these miRNAs may play an important role in the metastasis and may be a special biomarker for the lymph node metastasis.

Bioinformatic algorithms have played a key role in the discovery of miRNAs, prediction of target genes and miRNAome. TargetScan, PicTar and miRanda were commonly used to predict miRNA target genes. We identified thousands of target genes in which only a small fraction of miRNA are actually involved in the biological process. The algorithms mentioned above

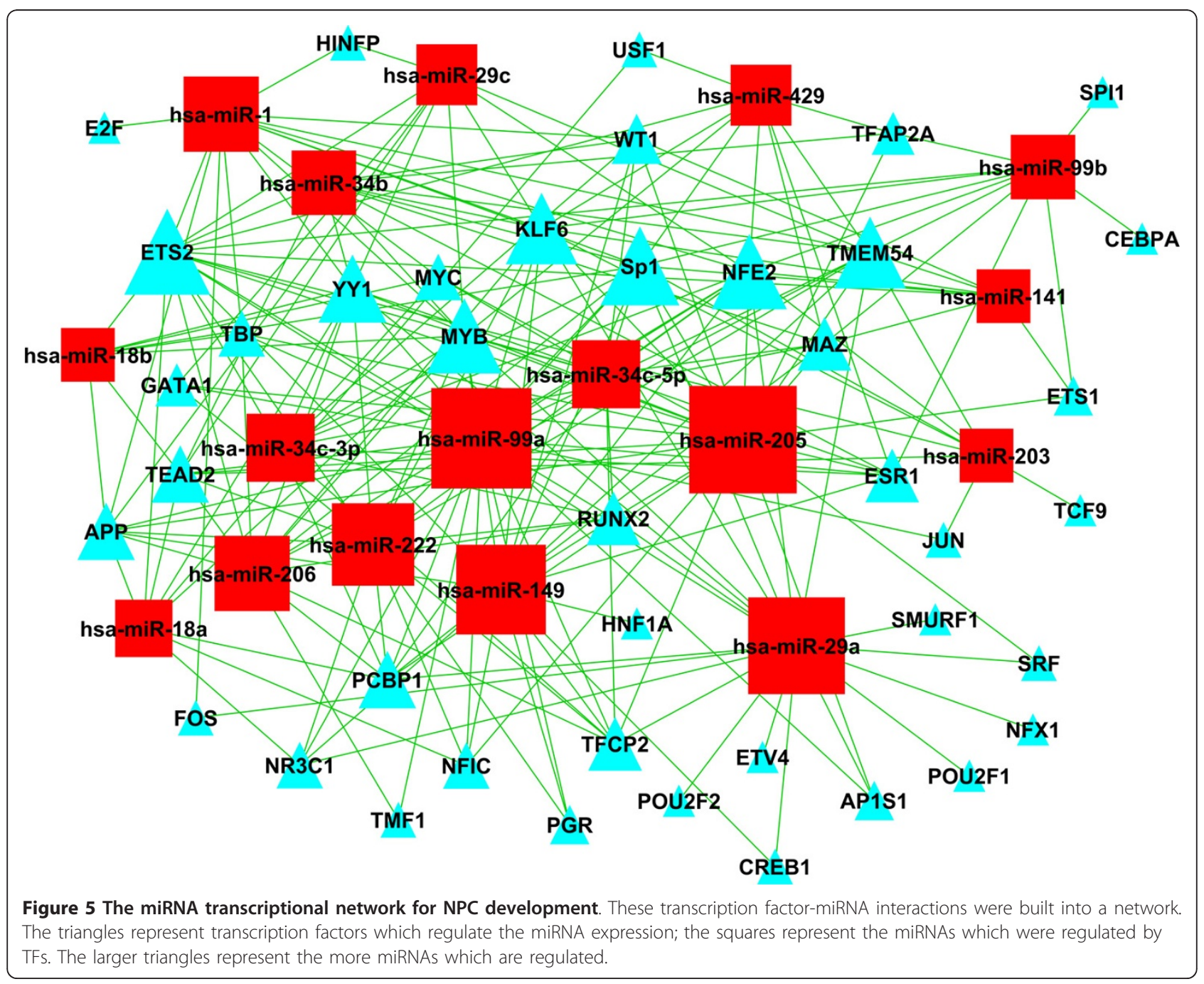


Table 6 TF-miRNAs-target genes feedback loop

\begin{tabular}{ccc}
\hline TF & miRNA & target gene \\
\hline ETS2 & hsa-miR-199b-5p & ETS2 \\
ETS2 & hsa-miR-203 & ETS2 \\
ETS2 & hsa-miR-222 & ETS2 \\
ETS2 & hsa-miR-335 & ETS2 \\
ETS2 & hsa-miR-429 & ETS2 \\
SP1 & hsa-miR-1 & SP1 \\
SP1 & hsa-miR-130a & SP1 \\
SP1 & hsa-miR-130b & SP1 \\
SP1 & hsa-miR-135a & SP1 \\
SP1 & hsa-miR-148b & SP1 \\
SP1 & hsa-miR-149 & SP1 \\
SP1 & hsa-miR-181c & SP1 \\
SP1 & hsa-miR-199b-5p & SP1 \\
SP1 & hsa-miR-203 & SP1 \\
SP1 & hsa-miR-206 & SP1 \\
SP1 & hsa-miR-32 & SP1 \\
SP1 & hsa-miR-335 & SP1 \\
SP1 & hsa-miR-429 & SP1 \\
SP1 & hsa-miR-486-3p & SP1 \\
KLF6 & hsa-miR-181c & KLF6 \\
\hline
\end{tabular}

showed limited consistency among predicted targets. It is necessary to identify the confirmed target genes which are actually related to the miRNA regulation function. In this study, the thousands of target genes were refined by screening with the nasopharyngeal- specifically expressed genes. Another data reduction strategy is to compare the miRNA expression and cDNA expression data and select the miRNA which is consistent with the cDNA expression [48]. The cDNA expression data (GSE12452) were downloaded from the publicly-accessible National Center for Biotechnology Information-Gene Expression Omnibus (GEO). The data reduction strategies narrowed down the target miRNA list and promoted the accuracy of high-throughput technology and bioinformatics. Using these data reduction strategy, we narrowed down the miRNAs list, showing that $\mathrm{miR}-29 \mathrm{a} / \mathrm{c}, \mathrm{miR}-34 \mathrm{~b} / \mathrm{c}, \mathrm{miR}-429, \mathrm{miR}-203$, miR-222, miR-1/206, miR-141, miR-18a/b, miR-544, miR-205 and miR-149 may be the most important modulator during the development of NPC. The expressions of these miRNAs were also validated using real-time RT-PCR.

It is estimated that $1-4 \%$ of genes in the human genome encode miRNAs and a single miRNA can regulate as many as 200 mRNAs [49]. The expression of miRNAs can be activated or repressed by transcription factors (TFs), which therefore can serve as upstream regulators of miRNAs. In recent years, many researchers have attempted to understand how miRNAs act to regulate target genes and what their roles are in various diseases.
However, the study of miRNAs regulation by TFs (TFmiRNA regulation) has been relatively limited. We reported previously that miRNAs and TFs may cooperate to regulate target gene expression. In addition, miRNAs and TFs can form feedback or feed-forward loops, which play critical roles in various biological processes. For example, ETS2 induces expression of miR-7 which, in turn, suppresses the expression of ETS2 activity. Increasing evidence suggests that aberrant regulation of miRNAs by TFs can cause diseases. Therefore, TFmiRNA regulation is one of the most important aspects of the study of both miRNAs and TFs. In this study, we bioinformatically predicted twenty-one TFs, seven of which may be the key regulator of the miRNA expression. This study provides an initial valuable data set for the miRNA regulation and its function.

\section{Conclusions}

The goal of profiling miRNA expression in this study is to discover the specific miRNAs in which influence the development of NPC. We found that miR-29a/c, miR34b, miR-34c-3p, miR-34c-5p, miR-429, miR-203, miR222, miR-1/206, miR-141, miR-18a/b, miR-544, miR-205 and miR-149 may play important roles in this respect. Merging the mRNA expression array and building an integrated molecular net-work associated with NPC enables us to gain a better understanding of the overall pathology. The transcription factors that including ETS2, MYB, Sp1, KLF6, NFE2, PCBP1 and TMEM54 exert regulatory functions on the miRNA expression. Three of them were predicted to repress their own translation through a TF-miRNAs-gene feedback loop. Bioinformatics and data filtering strategy were combined to screen the miRNAs which may be the key modulator in the process of tumorigenesis.

\section{Additional material}

Additional file 1: Clinical features of patients. The information is available online.

\section{Acknowledgements}

This work was supported by national natural science foundation of China (project number 81000882); the National Training and Research Base for Talents of principles of carcinogenesis foundation (111 project: 111-2-12); Hunan Natural Science Foundation (10JJ7003) and Facilities Sharing Fund of Central South University (ZKJ2010006).

\section{Author details}

${ }^{1}$ Cancer Research Institute, Key Laboratory of Carcinogenesis and Cancer Invasion of Ministry of Education, Key Laboratory of Carcinogenesis of Ministry of Health, Central South University, 110 Xiangya Road, Changsha, Hunan, 410078, P.R. China. ${ }^{2}$ The Li Ka Shing Institute of Health Sciences, Department of Orthopaedics \& Traumatology, Faculty of Medicine, The Chinese University of Hong Kong, Prince of Wales Hospital, Shatin, NT, Hong Kong. 


\section{Authors' contributions}

The work presented here was carried out as a collaboration between all authors. ZHL, LYZ and ZL carried out most experiments. HBY and XFG collected the biopies and the data. JJX, ZHL, ZL and GYL made contributions to design, analyze data and interpret data. JJX, ZHL, GL and XYL have been involved in drafting the manuscript. GYL gave most financial support. YFD and $\mathrm{CJ}$ collected and assembled the data. All the authors have given final approval to publish the manuscript.

\section{Competing interests}

The authors declare that they have no competing interests.

Received: 9 August 2011 Accepted: 19 January 2012

Published: 19 January 2012

\section{References}

1. Chou J, Lin YC, Kim J, You L, Xu Z, He B, Jablons DM: Nasopharyngeal carcinoma-review of the molecular mechanisms of tumorigenesis. Head Neck 2008, 30(7):946-963.

2. Lin JC, Liao SK, Lee EH, Hung MS, Sayion Y, Chen HC, Kang CC, Huang LS, Cherng JM: Molecular events associated with epithelial to mesenchymal transition of nasopharyngeal carcinoma cells in the absence of EpsteinBarr virus genome. J Biomed Sci 2009, 16:105.

3. Tao Y, Bidault F, Bosq J, Bourhis J: Distant metastasis of undifferentiated carcinoma of nasopharyngeal type. Onkologie 2008, 31(11):574-575.

4. Yoshizaki T, Ito M, Murono S, Wakisaka N, Kondo S, Endo K: Current understanding and management of nasopharyngeal carcinoma. Auris Nasus Larynx 2011

5. Baranwal S, Alahari SK: miRNA control of tumor cell invasion and metastasis. Int J Cancer 2009, 126(6):1283-1290.

6. Barker EV, Cervigne NK, Reis PP, Goswami RS, Xu W, Weinreb I, Irish JC, Kamel-Reid S: microRNA evaluation of unknown primary lesions in the head and neck. Mol Cancer 2009, 8:127.

7. Nugent M, Miller N, Kerin MJ: MicroRNAs in colorectal cancer: Function, dysregulation and potential as novel biomarkers. Eur I Surg Oncol 2011, 37(8):649-654.

8. Johnson SM, Grosshans H, Shingara J, Byrom M, Jarvis R, Cheng A, Labourier E, Reinert KL, Brown D, Slack FJ: RAS is regulated by the let-7 microRNA family. Cell 2005, 120(5):635-647.

9. Ma L, Reinhardt F, Pan E, Soutschek J, Bhat B, Marcusson EG, TeruyaFeldstein J, Bell GW, Weinberg RA: Therapeutic silencing of miR-10b inhibits metastasis in a mouse mammary tumor model. Nat Biotechnol 2010, 28(4):341-347.

10. Deng M, Tang H, Zhou Y, Zhou M, Xiong W, Zheng Y, Ye Q, Zeng X, Liao $Q$, Guo $X$, et al: miR-216b suppresses tumor growth and invasion by targeting KRAS in nasopharyngeal carcinoma. J Cell Sci 2011, 124(Pt 17):2997-3005.

11. Alajez NM, Lenarduzzi M, Ito E, Hui AB, Shi W, Bruce J, Yue S, Huang SH, Xu W, Waldron J, et al: MiR-218 suppresses nasopharyngeal cancer progression through downregulation of survivin and the SLIT2-ROBO1 pathway. Cancer Res 2011, 71(6):2381-2391.

12. Lu J, He ML, Wang L, Chen Y, Liu X, Dong Q, Chen YC, Peng Y, Yao KT, Kung HF, et al: MiR-26a inhibits cell growth and tumorigenesis of nasopharyngeal carcinoma through repression of EZH2. Cancer Res 2011, 71(1):225-233.

13. Ji $Y$, He $Y$, Liu L, Zhong $X$ : MiRNA-26b regulates the expression of cyclooxygenase-2 in desferrioxamine-treated CNE cells. FEBS Lett 2010, 584(5):961-967.

14. Li G, Wu Z, Peng Y, Liu X, Lu J, Wang L, Pan Q, He ML, Li XP: MicroRNA$10 \mathrm{~b}$ induced by Epstein-Barr virus-encoded latent membrane protein-1 promotes the metastasis of human nasopharyngeal carcinoma cells. Cancer Lett 2010, 299(1):29-36.

15. Wong TS, Man OY, Tsang CM, Tsao SW, Tsang RK, Chan JY, Ho WK, Wei WI, To VS: MicroRNA let-7 suppresses nasopharyngeal carcinoma cells proliferation through downregulating c-Myc expression. J Cancer Res Clin Oncol 2010, 137(3):415-422.

16. Zhang L, Deng T, Li X, Liu H, Zhou H, Ma J, Wu M, Zhou M, Shen S, Niu Z, et al: microRNA-141 is involved in a nasopharyngeal carcinoma-related genes network. Carcinogenesis 2010, 31(4):559-566.

17. Xia H, Ng SS, Jiang S, Cheung WK, Sze J, Bian XW, Kung HF, Lin MC: miR200a-mediated downregulation of ZEB2 and CTNNB1 differentially inhibits nasopharyngeal carcinoma cell growth, migration and invasion. Biochem Biophys Res Commun 2009, 391(1):535-541.

18. Wong AM, Kong KL, Tsang JW, Kwong DL, Guan XY: Profiling of EpsteinBarr virus-encoded microRNAs in nasopharyngeal carcinoma reveals potential biomarkers and oncomirs. Cancer 2011.

19. Barth S, Meister G, Grasser FA: EBV-encoded miRNAs. Biochim Biophys Acta 2011, 1809:631-640.

20. Chen SJ, Chen GH, Chen YH, Liu CY, Chang KP, Chang YS, Chen HC: Characterization of Epstein-Barr virus miRNAome in nasopharyngeal carcinoma by deep sequencing. PLoS One 2010, 5(9):pii: e12745.

21. Cho WC: Nasopharyngeal carcinoma: molecular biomarker discovery and progress. Mol Cancer 2007, 6:1.

22. Barth S, Meister G, Grasser FA: EBV-encoded miRNAs. Biochim Biophys Acta 2011, 1809(11-12):631-640.

23. Cho WC: MicroRNAs: potential biomarkers for cancer diagnosis, prognosis and targets for therapy. Int J Biochem Cell Biol 2010, 42(8):1273-1281.

24. Lo AK, To KF, Lo KW, Lung RW, Hui JW, Liao G, Hayward SD: Modulation of LMP1 protein expression by EBV-encoded microRNAs. Proc Natl Acad Sci USA 2007, 104(41):16164-16169.

25. Chen HC, Chen GH, Chen YH, Liao WL, Liu CY, Chang KP, Chang YS, Chen SJ: MicroRNA deregulation and pathway alterations in nasopharyngeal carcinoma. Br J Cancer 2009, 100(6):1002-1011.

26. Sengupta S, den Boon JA, Chen IH, Newton MA, Stanhope SA, Cheng YJ, Chen CJ, Hildesheim A, Sugden B, Ahlquist P: MicroRNA 29c is downregulated in nasopharyngeal carcinomas, up-regulating mRNAs encoding extracellular matrix proteins. Proc Natl Acad Sci USA 2008, 105(15):5874-5878

27. Li T, Chen JX, Fu XP, Yang S, Zhang Z, Chen Kh H, Li Y: microRNA expression profiling of nasopharyngeal carcinoma. Oncol Rep 2011, 25(5):1353-1363

28. Olaru AV, Selaru FM, Mori Y, Vazquez C, David S, Paun B, Cheng Y, Jin Z, Yang J, Agarwal R, et al: Dynamic changes in the expression of MicroRNA-31 during inflammatory bowel disease-associated neoplastic transformation. Inflamm Bowel Dis 2010, 17(1):221-231.

29. Bandyopadhyay S, Bhattacharyya M: PuTmiR: a database for extracting neighboring transcription factors of human microRNAs. BMC Bioinformatics 2010, 11:190.

30. Megraw M, Baev V, Rusinov V, Jensen ST, Kalantidis K, Hatzigeorgiou AG: MicroRNA promoter element discovery in Arabidopsis. RNA 2006, 12(9):1612-1619.

31. Cho WC: MicroRNAs in cancer - from research to therapy. Biochim Biophys Acta 2010, 1805(2):209-217.

32. Mao YP, Li WF, Chen L, Sun Y, Liu LZ, Tang LL, Cao SM, Lin AH, Hong MH, Lu TX, et al: [A clinical verification of the Chinese 2008 staging system for nasopharyngeal carcinoma]. Ai Zheng 2009, 28(10):1022-1028.

33. Sun Y, Ma J: [Comment for the Chinese 2008 staging system for nasopharyngeal carcinoma]. Ai Zheng 2009, 28(10):1016-1021.

34. Clarke R, Ressom HW, Wang A, Xuan J, Liu MC, Gehan EA, Wang Y: The properties of high-dimensional data spaces: implications for exploring gene and protein expression data. Nat Rev Cancer 2008, 8(1):37-49.

35. Wright GW, Simon RM: A random variance model for detection of differential gene expression in small microarray experiments. Bioinformatics 2003, 19(18):2448-2455.

36. Yang $\mathrm{H}$, Crawford N, Lukes L, Finney R, Lancaster M, Hunter KW: Metastasis predictive signature profiles pre-exist in normal tissues. Clin Exp Metastasis 2005, 22(7):593-603.

37. Prieto C, Risueno A, Fontanillo C, De las Rivas J: Human gene coexpression landscape: confident network derived from tissue transcriptomic profiles. PLoS One 2008, 3(12):e3911.

38. Vermeirssen V, Barrasa MI, Hidalgo CA, Babon JA, Sequerra R, DoucetteStamm L, Barabasi AL, Walhout AJ: Transcription factor modularity in a gene-centered $C$. elegans core neuronal protein-DNA interaction network. Genome Res 2007, 17(7):1061-1071.

39. Joung JG, Hwang KB, Nam JW, Kim SJ, Zhang BT: Discovery of microRNAmRNA modules via population-based probabilistic learning. Bioinformatics 2007, 23(9):1141-1147.

40. Chen SJ, Chen GH, Chen YH, Liu CY, Chang KP, Chang YS, Chen HC: Characterization of Epstein-Barr virus miRNAome in nasopharyngeal carcinoma by deep sequencing. PLoS One 2010, 5(9). 
41. Ramsingh G, Koboldt DC, Trissal M, Chiappinelli KB, Wylie T, Koul S, Chang LW, Nagarajan R, Fehniger TA, Goodfellow P, et al: Complete characterization of the microRNAome in a patient with acute myeloid leukemia. Blood 2010, 116(24):5316-5326.

42. Park SY, Lee JH, Ha M, Nam JW, Kim VN: miR-29 miRNAs activate $p 53$ by targeting p85 alpha and CDC42. Nat Struct Mol Biol 2009, 16(1):23-29.

43. Xu H, Cheung IY, Guo HF, Cheung NK: MicroRNA miR-29 modulates expression of immunoinhibitory molecule B7-H3: potential implications for immune based therapy of human solid tumors. Cancer Res 2009, 69(15):6275-6281.

44. Chow TF, Mankaruos M, Scorilas A, Youssef Y, Girgis A, Mossad S, Metias S, Rofael Y, Honey RJ, Stewart R, et al: The miR-17-92 cluster is over expressed in and has an oncogenic effect on renal cell carcinoma. J Urol 2009, 183(2):743-7451.

45. Diosdado B, van de Wiel MA, Terhaar Sive Droste JS, Mongera S, Postma C, Meijerink WJ, Carvalho B, Meijer GA: MiR-17-92 cluster is associated with 13q gain and c-myc expression during colorectal adenoma to adenocarcinoma progression. Br J Cancer 2009, 101(4):707-714.

46. Adams BD, Cowee DM, White BA: The role of miR-206 in the epidermal growth factor (EGF) induced repression of estrogen receptor-alpha (ERalpha) signaling and a luminal phenotype in MCF-7 breast cancer cells. Mol Endocrinol 2009, 23(8):1215-1230.

47. Ai J, Zhang R, Li Y, Pu J, Lu Y, Jiao J, Li K, Yu B, Li Z, Wang R, et al: Circulating microRNA-1 as a potential novel biomarker for acute myocardial infarction. Biochem Biophys Res Commun 2009, 391(1):73-77.

48. Cho JH, Gelinas R, Wang K, Etheridge A, Piper MG, Batte K, Dakhallah D, Price J, Bornman D, Zhang $S$, et al: Systems biology of interstitial lung diseases: integration of mRNA and microRNA expression changes. BMC Med Genomics 2011, 4:8.

49. Shalgi R, Lieber D, Oren M, Pilpel Y: Global and local architecture of the mammalian microRNA-transcription factor regulatory network. PLOS Comput Biol 2007, 3(7):e131.

\section{Pre-publication history}

The pre-publication history for this paper can be accessed here: http://www.biomedcentral.com/1755-8794/5/3/prepub

doi:10.1186/1755-8794-5-3

Cite this article as: Luo et al: An in silico analysis of dynamic changes in microRNA expression profiles in stepwise development of nasopharyngeal carcinoma. BMC Medical Genomics 2012 5:3.

\section{Submit your next manuscript to BioMed Central and take full advantage of:}

- Convenient online submission

- Thorough peer review

- No space constraints or color figure charges

- Immediate publication on acceptance

- Inclusion in PubMed, CAS, Scopus and Google Scholar

- Research which is freely available for redistribution

Submit your manuscript at www.biomedcentral.com/submit 